\title{
Recombination nodules, chiasmata and crossing-over in the nucleolus organizing short arm of Allium fistulosum
}

\author{
G. H. Jones and \\ S. M. Albini
}

\author{
Department of Genetics, University of Birmingham, \\ Birmingham B15 2TT, U.K.
}

\begin{abstract}
The relationship of late recombination nodules (RNs) to chiasmata and crossing-over was examined in a short defined chromosome region of Allium fistulosum. The nucleolus organising region (NOR) occupies a subterminal position on the short arm of the most acrocentric bivalent of this species and is easily recognised in surface spread pachytene synaptonemal complexes (SCs) and at metaphase I and anaphase I. Some of the plants examined had heteromorphic nucleolus organising short arms, involving deletion of the NOR from one homologue. This system allowed comparison of late RN frequency in pachytene SCs with metaphase-I chiasma frequency, and also with crossover frequency based on first division segregation of the NOR deletion. Despite general and localised deficits of late RNs compared to chiasmata and crossovers in $\boldsymbol{A}$. fistulosum, the frequency of late RNs in this defined short region did not differ significantly from the other estimates of recombination frequency.
\end{abstract}

\section{INTRODUCTION}

Nodule structures, generally referred to as recombination nodules (RNs), have been found associated with synaptonemal complexes (SCs) in a wide range of organisms having chiasmate meiosis (von Wettstein et al., 1984). Recently, it has been increasingly recognised that RNs fall into two classes, principally defined according to their chronology of appearance in meiotic prophase I, and termed early and late RNs* (Carpenter, 1987).

Although early and late RNs generally differ to some degree in respect of chronology and/or morphology, these differences are usually quantitative rather than qualitative and seldom unambiguously differentiate the two nodule classes. Fortunately, some species do allow consistent differentiation of early and late RNs on the basis of morphology, as in Drosophila melanogaster which has ellipsoid early RNs and spherical late RNs (Carpenter, 1979), or on the basis of distinct non-overlapping chronologies as in Allium cepa and $A$. fistulosum (Albini and Jones, 1987). These

\footnotetext{
* Elsewhere these structures have been called early and late nodules (Jones and Albini, in press) and the early category have also been termed zygotene nodules $(\mathrm{ZN})$ in Allium species where they are confined to that stage (Albini and Jones, 1987). In this paper we conform with the more established terminology and refer to them as early RNs and late RNs.
}

species are therefore especially suitable for investigating the relationships of nodules to recombination events, as extensively documented for $D$. melanogaster (Carpenter, 1984).

Early and late RNs have recently been investigated in A. cepa and A. fistulosum with particular reference to their meiotic functions. The characteristics of early RNs suggest that they may be directly involved in matching homologous sequences during chromosome pairing (Albini and Jones, 1987 ) and they may possibly mediate gene conversion (Carpenter, 1987). Despite deficits in the numbers of late RNs compared to chiasmata, the positional distributions of late RNs correlate remarkably closely with the contrasting chiasma distributions of these Allium species (Albini and Jones, in press), supporting the hypothesis that late RNs are directly involved in reciprocal recombination events (Carpenter, 1979, 1987). However, this is a general correspondence based on comparisons of the cumulative late $\mathrm{RN}$ and chiasma distributions of all eight chromosome pairs present in these species. Thus, for instance, proximally localised chiasmata and late RNs are general, but not invariable, characteristics of all eight bivalents of $A$. fistulosum.

More stringent tests of this correspondence require parallel observations on late $\mathrm{RNs}$ and chiasmata/crossovers in short defined chromo- 
some regions. Just such a test system is provided by the short nucleolus organizing (NO) arm of bivalent 8 of $A$. fistulosum. In $A$. fistulosum, as in other species belonging to the cepa section of the genus Allium, the NO bivalent is the most asymmetrical (acrocentric) member of the complement, all other bivalents being metacentric or sub-metacentric (Vosa, 1976). It is therefore easily distinguished by its arm ratio, when the centromere is discernible, as well as by its possession of a nucleolus organising region (NOR) which identifies it at pachytene through its association with nucleolar material, and at later meiotic stages either by its satellited short arm in orcein stained preparations or by its silver staining reaction. In one particular clone of $A$. fistulosum, the NOR bivalent is heteromorphic as a result of a deletion of part of the short arm including the NOR. This feature permits a direct assessment of crossing-over in this bivalent arm, as well as the incidence of chiasmata and late RNs.

\section{MATERIALS AND METHODS}

The A. fistulosum material used in this study (designated Leiden 767) was donated by Dr B. Ford-Lloyd, Department of Plant Biology, University of Birmingham. The bulbs were grown in pots in a glasshouse and given supplementary lighting (18 hour days) to stimulate the production of inflorescences in January-March. Flowering in natural daylight occurred in March-April sometimes followed by a second flowering in late summer.

For the study of SCs and late RNs, meiocytes were surface-spread using the method developed by Albini and Jones $(1984,1987)$. The spread nuclei were stained with ethanolic phosphotungstic acid (PTA), as described previously (Albini and Jones, 1987) and suitable nuclei were transferred to grids for examination in a Philips EM301 electron microscope.

For light microscopical studies of meiotic NORs the procedure adopted by Loidl and Greilhuber (1983) was used. Flower buds at the appropriate stage were fixed in Carnoy's fluid (6 ethanol, 3 chloroform, 2 glacial acetic acid) overnight. Pollen mother cells were squashed in 45 per cent acetic acid, checked for stage by phase contast, and then coverslips were removed after freezing the slides in liquid nitrogen. The slides were allowed to air dry and stored overnight. Silver staining was carried out by placing one drop of freshly prepared 50 per cent silver nitrate solution under a nylon gauze coverslip (Loidl and Jones, 1986) and incubating in a moisture chamber at $60^{\circ} \mathrm{C}$ for $30-35$ minutes. After checking the progress of staining under the microscope, the coverslip and stain were washed off in running tap water and the slides rinsed in distilled water for two minutes before air drying and mounting a fresh glass coverslip in Euparal Vert.

Chiasma observations were made on acetic orcein stained squash preparations of pollen mother cells at metaphase I of meiosis.

\section{RESULTS}

\section{The nucleolus organizing bivalent at pachytene}

The nucleolus organizing bivalent was clearly identifiable in surface spread pachytene nuclei of $A$. fistulosum by its acrocentric nature, and by the characteristic appearance of the nucleolus organizing region, in particular its association with remnants of nucleolar material and/or disturbances of SC formation (figs. 1 and 2). The mean length of this bivalent's SC, estimated from plants with homomorphic NORs in which pairing failures and disturbances were minimal, was $85 \cdot 55 \mu \mathrm{m}$ (11 nuclei). Both its mean and modal rank positions placed it as the fourth longest SC in the complement. The mean centromere index of this $\mathrm{SC}$ was $0 \cdot 244$ and its arm ratio was consequently $3 \cdot 1: 1$. The mean length of the nucleolus organising short arm SC was $20.87 \mu \mathrm{m}$. In contrast to $A$. cepa, in which the nucleolus was often well preserved in surface spreads, the nucleolus was often dispersed in $A$. fistulosum and in these cases only remnants of nucleolar material were found in association with the NOR. This conferred the advantage that the SC and any unpaired axial cores (ACs) could be followed with relative ease whereas intact nucleoli generally obscured associated SCs. As far as could be ascertained from the distribution of nucleolar material, the NOR occupies about 25 per cent of the short arm in A. fistulosum and is subterminally located (see fig. 3 ).

\section{NOR hetermorphism}

Certain plants of $\boldsymbol{A}$. fistulosum showed a marked heteromorphism of the nucleolus organizing short arm, which was detectable at the EM level in pachytene surface spread SCs (fig. 2) and also in silver stained squash preparations of metaphase I and anaphase I (figs. 4 and 5). In surface spreads, one homologue (measured through paired and unpaired regions) was consistently shorter than 


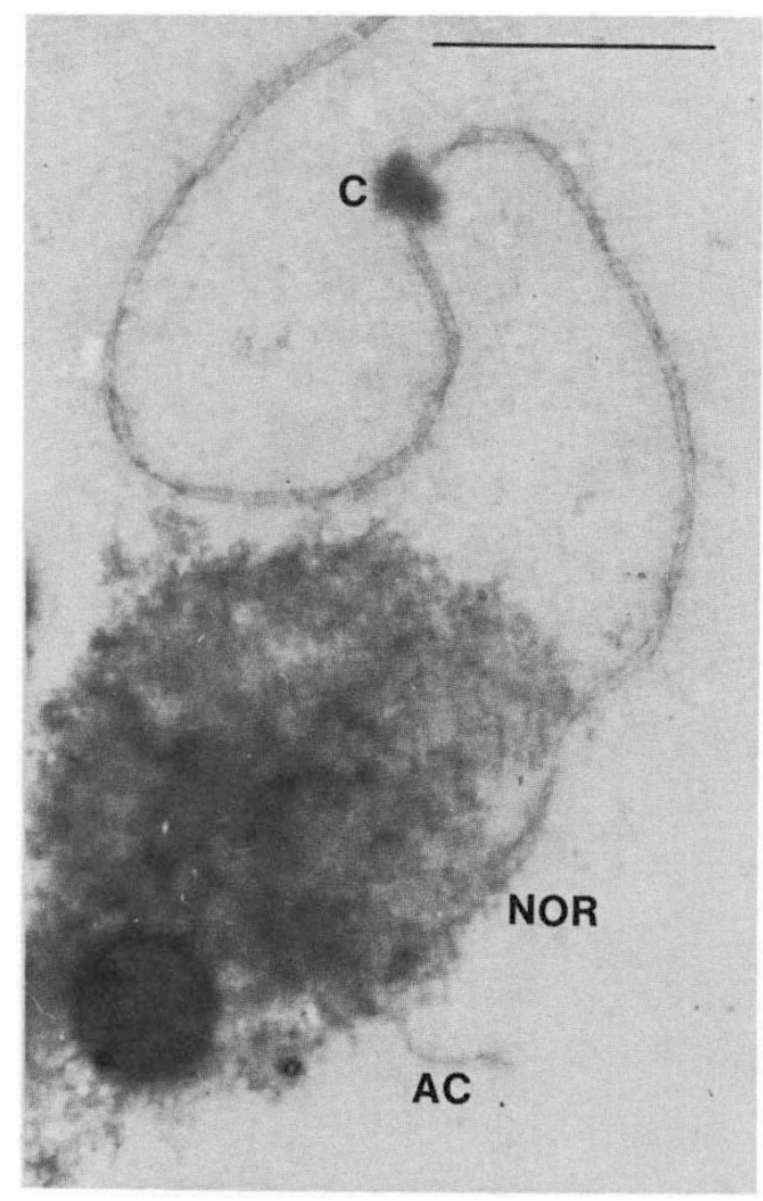

Figure 1 Electron micrograph of part of a surface spread SC of a homomorphic NO bivalent of A. fistulosum. The short arm is fully paired between the centromere (C) and the nucleolus organizer (NOR). The extreme distal end of the bivalent, beyond the NOR, is evidently unpaired, judging by the single projecting axial core $(\mathrm{AC})$. Bar $=5 \mu \mathrm{m}$.

the other, the average length difference indicating that a deletion of about 30 per cent of the short arm had occurred. Nucleolar material was confined to the longer AC suggesting that the deletion included the entire NOR, and this was confirmed by the absence of silver staining from one homologue at metaphase I (fig. 4(a)). A segmental deletion of part of the NO short arm has been previously reported in the cultivated variety "Kujo-negi" of A. fistulosum (Zen, 1961). However in the absence of information on silver staining and meiotic pairing behaviour it is difficult to compare that deletion to the present case.

In contrast to normal homomorphic bivalents, heteromorphic bivalents showed extensive but variable asynapsis of the short arm. In about 30 per cent of nuclei the short arm was completely unpaired (fig. 2(a)), while the remainder showed partial pairing extending from the centromere for variable distances but always ceasing proximally to the NOR (fig. 2(b)). The region distal to the NOR was always unpaired in heteromorphic nuclei.

\section{RNs in NO bivalents}

Well spread PTA-stained pachytene SCs, which were relatively free of chromatin, showed clear ellipsoid or spherical nodules (late RNs) associated with the central region or, sometimes, overlying or to one side of one lateral element. The general characteristics of these late RNs in $A$. fistulosum are fully described elsewhere (Albini and Jones, in press). Seventy-three per cent of 

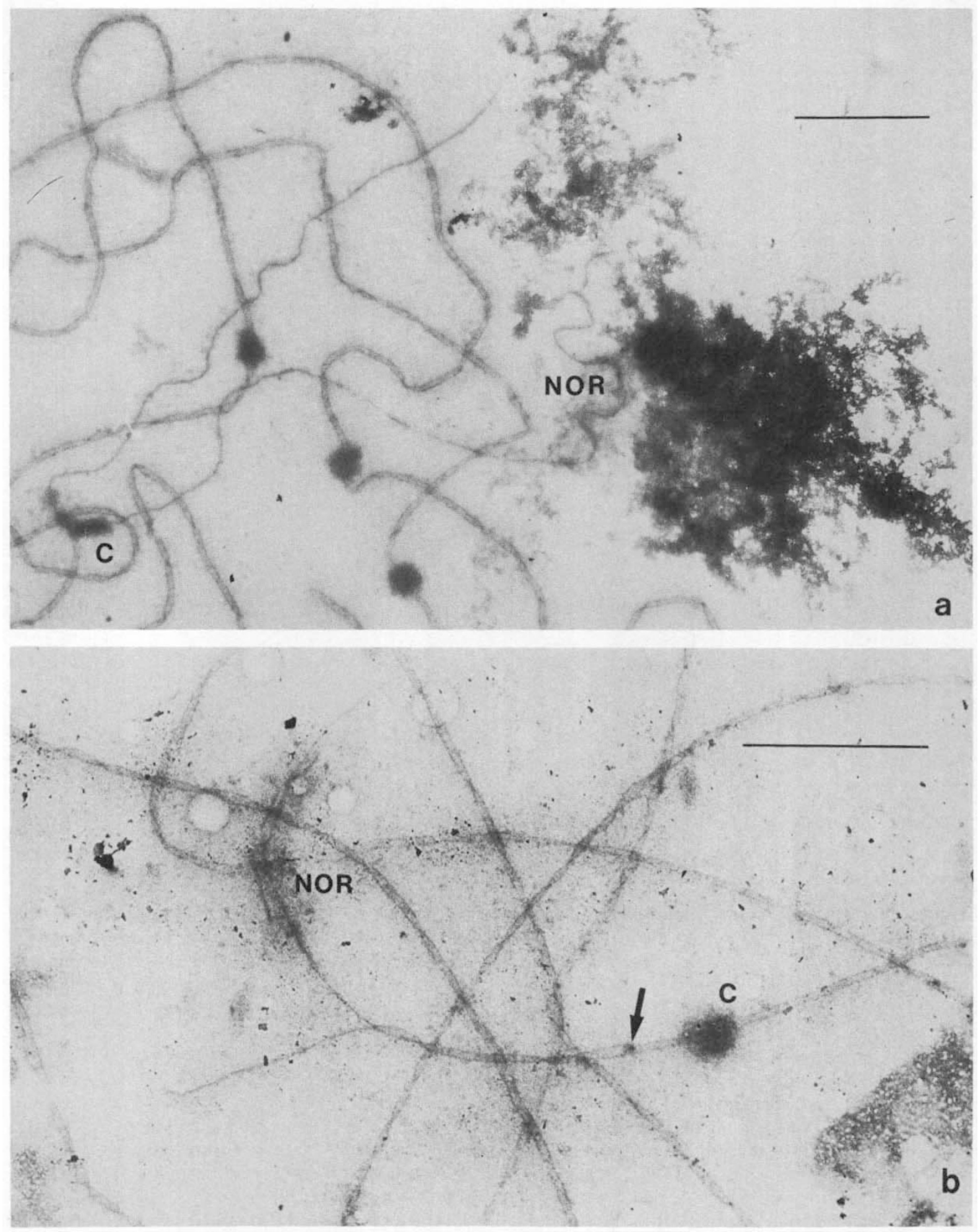

Figure 2 Electron micrographs of surface spread SCs of A. fistulosum showing unpaired (a) and partially paired (b) hetermorphic NO short arms. A prominent RN (arrowed) is present in the partially paired short arm SC. In both cases the nucleolus has been dispersed and remnants of nucleolar material are associated with the nucleolus organizer $(\mathrm{NOR}) . \mathrm{C}=\mathrm{centromere} ; \mathrm{Bars}=5 \mu \mathrm{m}$. 
NOR

Figure 3 A schematic diagram of the nucleolus organizing chromosome of A. fistulosum. $\mathrm{C}=$ centromere; $\mathrm{NOR}=$ nucleolus organizing region.

homomorphic NO short arms contained a single RN (fig. 2(b)), which was invariably located between the centromere and the NOR. Twenty-five heteromorphic NO bivalents were analysed from clear well spread pachytenes in which RNs were clearly visible in most SCs. Of these, 8 had completely unpaired short arms and of the remaining 17 with partially paired short arms, 9 had a single RN each and 8 lacked RNs (table 1). A relationship between late RNs and the extent of SC in the NO short arm is evident from these data; seven of the ten longest $\mathrm{SC}$ s possess a late RN while six of the ten shortest SCs lack RNs. However, the relationship is far from absolute since, for example, the second longest SC lacks a RN and the second shortest SC has one.

\section{Metaphase I chiasmata}

Homomorphic NO bivalents typically formed a single proximal chiasma in the long-arm, sometimes accompanied by a second interstitial or distal chiasma, and very regularly $(0 \cdot 95-1 \cdot 00)$ a single chiasma in the short NO arm. These short arm chiasmata could not be readily assigned locations (proximal, interstitial or distal) due to the relative

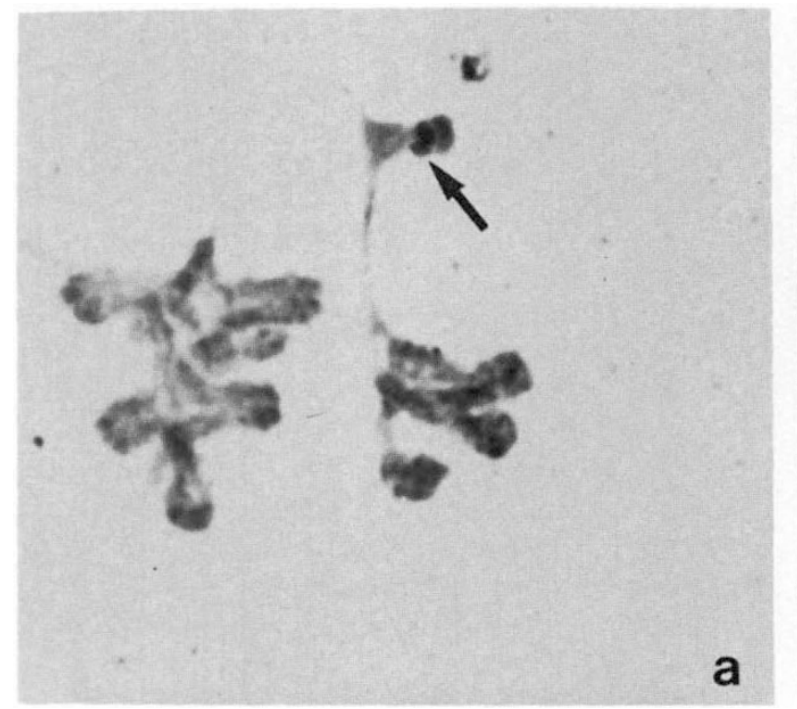

Table 1 The extent of pachytene SC formation and the RN status (+or-) of 17 partially paired nucleolus organising (NO) short arms. A further 8 nuclei had completely unpaired NO short arms

\begin{tabular}{ccc}
\hline \multirow{2}{*}{ Nucleus } & Extent of SC $(\mu \mathrm{m})$ & RNs \\
\cline { 2 - 3 } 1 & 1.89 & - \\
2 & $2 \cdot 63$ & - \\
3 & $2 \cdot 76$ & + \\
4 & 3.40 & - \\
5 & $4 \cdot 43$ & + \\
6 & $5 \cdot 29$ & - \\
7 & $5 \cdot 36$ & - \\
8 & 5.60 & + \\
9 & $5 \cdot 70$ & - \\
10 & $7 \cdot 35$ & - \\
11 & 8.51 & + \\
12 & 8.84 & + \\
13 & 9.41 & - \\
14 & 10.30 & + \\
15 & 13.22 & + \\
16 & 14.75 & + \\
17 & 16.21 & + \\
\hline
\end{tabular}

shortness of the arm; the entire short arm is somewhat shorter than the proximal zone $\left(\frac{1}{3}\right)$ of the long arm. In marked contrast, heteromorphic short arms showed a much reduced chiasma frequency $\left(\frac{38}{81}=\right.$ $0 \cdot 47)$. However, the long arms of hetermorphic NO bivalents showed normal chiasma frequencies, comparable to those of homomorphic bivalents. The deletion heterozygote studied by Zen (1961) showed a much higher short arm chiasma frequency $(0 \cdot 80)$, which suggests that the deletion was different to the present case.

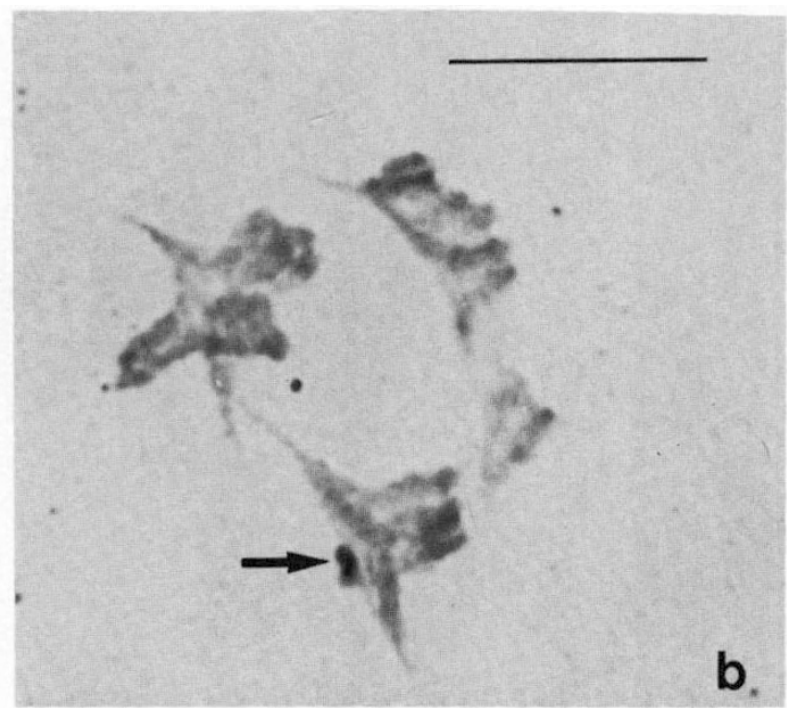

Figure 4 Silver stained metaphase I bivalents of A. fistulosum showing achiasmate (a) and chiasmate (b) NO short arms. The silver stained NOR (arrowed) is clearly confined to only one homologue. Bar $=10 \mu \mathrm{m}$. 
First division NOR segregation: crossover frequency

Cytological bivalent hetermorphisms can be used to directly assay the recombination frequencies of chromosome regions proximal to the markers (e.g., Brown and Zohary, 1955; Orellana and Giraldez, 1981). This method is simple and direct when the region being assayed is short enough not to permit more than one chiasma. When 2 or more chiasmata may occur the expectations are more complex since the proportions of equational segregation resulting from $0,1,2,3,4$ chiasmata follow the series 0,1 , $\frac{1}{2}, \frac{3}{4}, \frac{5}{8}$ of which the limiting value is $\frac{2}{3}$ reached when the chiasma frequency is very high (Mather, 1935). As the NO short arm is physically very short, it is reasonable to suppose that it can form a maximum of one chiasma and indeed cytological observations confirm this.

Forty-three per cent $\left(\frac{22}{51}\right)$ of silver stained first anaphases showed equational segregation of the
NOR (fig. 5(a)) and 57 per cent $\left(\frac{29}{51}\right)$ showed reductional segregation (fig. 5(b)). Silver stained dyads (pairs of interphase nuclei resulting from the first meiotic division) also give information on the relative frequencies of equational and reductional segregation of the NOR since nuclei containing NORs form discrete nucleoli while nuclei lacking NORs form scattered micronucleoli (fig. 6). Because dyads are much more frequent than first anaphase stages it was possible to sample many more with relative ease. In a sample of 100 dyads, 46 showed equational NOR segregation and 54 showed reductional segregation. Since, as argued earlier, the NO short arm forms a maximum of one chiasma, these equational segregation frequencies translate directly into crossover frequencies for the region between the centromere and the NOR. Chiasmata do not occur in the short region distal to the NOR since this region was never observed to pair at pachytene in heterozygotes.
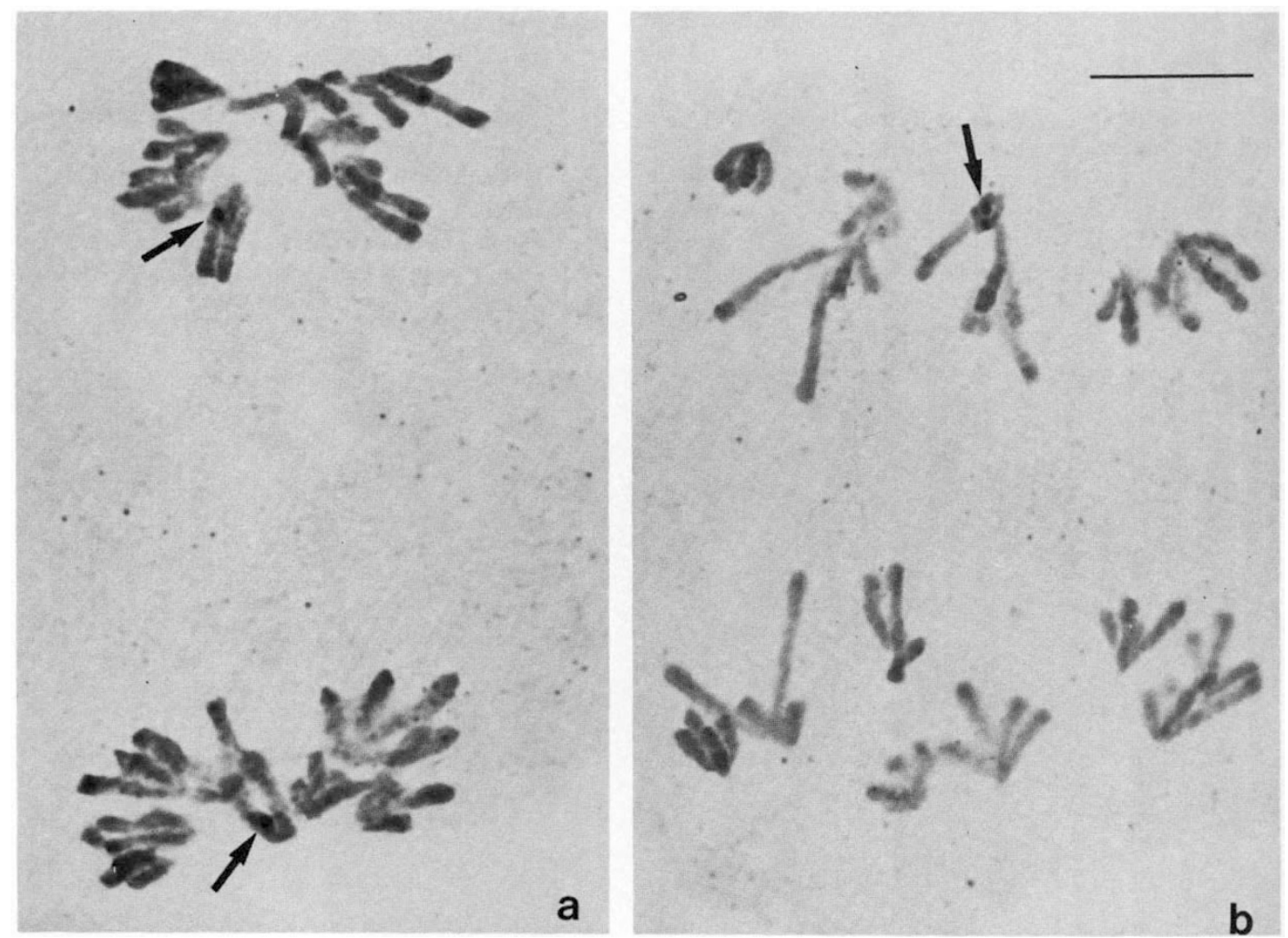

Figure 5 Silver stained anaphase I nuclei of A. fistulosum showing equational (a) and reductional (b) separation of the NOR (arrowed), $B a r=10 \mu \mathrm{m}$ ). 

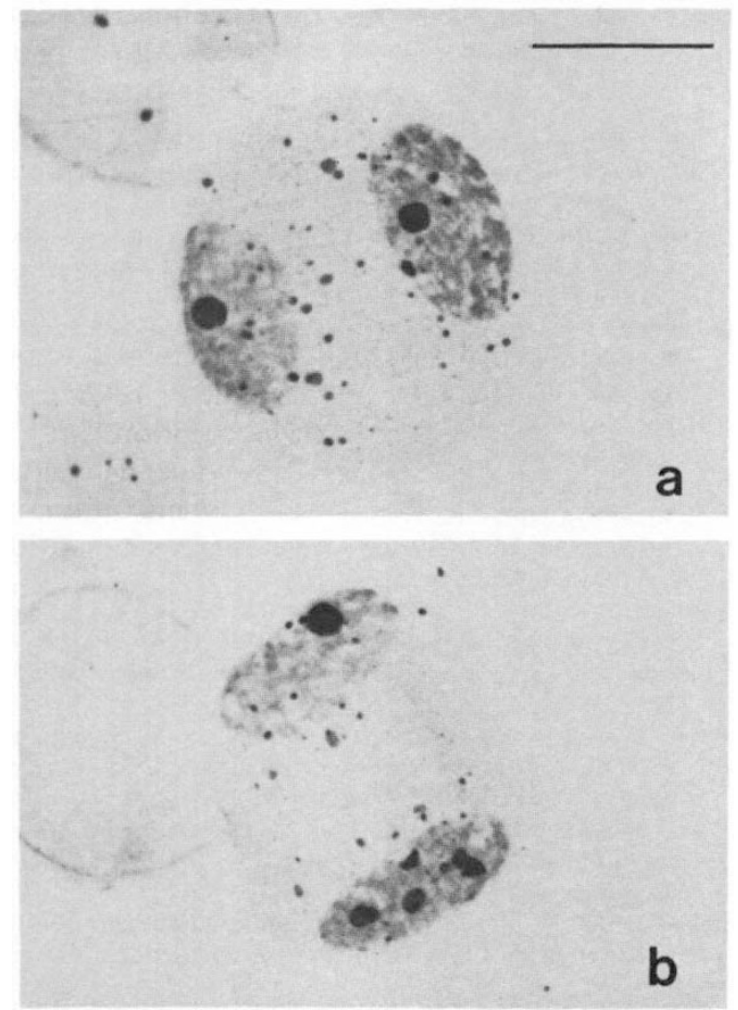

Figure 6 Silver stained dyads of A. fistulosum showing either two discrete nucleoli (a), the result of equational NOR segregation, or one discrete nucleolus and one nucleus with scattered micronucleoli (b), resulting from reductional NOR segration. Bar $=10 \mu \mathrm{m}$.

\section{The relationship of late $R N$ s to chiasmata and crossovers}

Comparative data on the frequencies of late (pachytene) RNs, metaphase I chiasmata and first division NOR segregation for the short arm of bivalent 8 are summarised in table 2 . It is evident from this table that the various light microscopical assessments of recombination (metaphase I chiasmata and anaphase $\mathrm{I} /$ interkinesis NOR segregation) show a close correspondence $\left(\chi_{(2)}^{2}=0 \cdot 187\right.$; $P>0 \cdot 90)$. Consequently these sources can be regarded as providing identical information and combined for testing against the pachytene RN data. A further test shows that the late RN frequency of the short arm does not differ significantly from the combined recombination frequency of this region $\left(\chi_{(1)}^{2}=0 \cdot 857, P>0 \cdot 30\right)$.
Table 2 Summary of data on presence (+) versus absence (-) of various light microscopical assessments of recombination (1-3) and RNs (4) in the NO short arm of A. fistulosum

\begin{tabular}{|c|c|c|c|}
\hline & + & - & \\
\hline 1. Metaphase I Chiasmata & 38 & $43)$ & \multirow{3}{*}{$\chi_{(2)}^{2}=0.187 ; P>0.9$} \\
\hline $\begin{array}{l}\text { 2. Anaphase I NOR Segre- } \\
\text { gation }\end{array}$ & 22 & $29\}$ & \\
\hline 3. Dyad NOR Segregation & 46 & 54 & \\
\hline $\begin{array}{l}\text { Combined "Recombi- } \\
\text { nation" }\end{array}$ & 106 & $126)$ & \multirow{2}{*}{$\chi_{(1)}^{2}=0.857 ; P>0.3$} \\
\hline 4. Late RNs & 9 & 16] & \\
\hline
\end{tabular}

\section{DISCUSSION}

A. fistulosum pachytene SCs show an overall deficiency of late RNs compared to chiasmata of about 25 per cent (Albini and Jones, in press). It appears that this deficiency is evenly distributed across the genome, since homomorphic NO short arms show an almost identical shortfall of nodules (chiasmata $0 \cdot 95-1 \cdot 00$, RNs $0 \cdot 75$ ). It is therefore reasonable to expect hetermorphic NO arms to show an equivalent deficiency of late RNs compared to chiasmata. This is in fact evident from the data since the mean late $\mathrm{RN}$ frequency of heteromorphic arms $(0 \cdot 360)$ is proportionally less than the combined recombination frequency $(0.456)$ to about the same extent. Nevertheless, despite this deficiency of late RNs, the heteromorphic NO short arm late RN frequency is not significantly less than the combined recombination frequency. While there is undoubtedly a consistent deficiency of RNs in A. fistulosum pachytene SCs, its magnitude is relatively small, and late RNs are therefore reasonably accurate indicators of reciprocal recombination events in this species.

By examining a short defined region $(\sim 2$ per cent) of the A. fistulosum genome, this comparative study has produced further convincing evidence that late RNs occur at the sites of reciprocal meiotic recombination. Comparable evidence is available only from parallel observations on very short chromosomes such as the microchromosomes of birds, and vertebrate sex chromosomes. In the domestic fowl single apparently obligate RNs appear in each of the short microchromosome SCs and also in the ZW sex bivalent where, additionally, the RNs are localised very near the distal end of the paired segment (Rahn and Solari, 1986). However, there is no equivalent data on genetical crossovers in the fowl and cytological observations of their chiasmata are uncertain due to the extremely small size of the microchromosomes and the extremely distal location of the $\mathrm{ZW}$ association. 
In both man and mouse about 75 per cent of XY SCs contain usually just one RN at pachytene (Holm and Rasmussen, 1983; Glamann, 1986) whereas cytological, genetical and molecular studies of XY recombination seems to indicate obligate single crossovers in these bivalents (Evans et al., 1982; Keitges et al., 1985; Rouyer et al., 1986). These discrepancies may be attributed either to transience of RNs (Glamann, 1986) or to absence of recombination in about 25 per cent of XY pairs, resulting in univalence (Hulten, 1974) which in turn results in spermatocyte degeneration (Burgoyne and Baker, 1984). The A. fistulosum comparison presented in this paper shows many parallels with these studies, and shows that the correspondence of late RNs to genetical recombination extends to a relatively short region within a very large chromosome.

\section{REFERENCES}

ALBINI, S. M. AND JONES, G. H. 1984. Synaptonemal complex associated centromeres and recombination nodules in plant meiocytes prepared by an improved surface-spreading technique. Exp. Cell Res., 155, 558-592.

ALBINI, S. M. AND JONES, G. H. 1987. Synaptonemal complex spreading in Allium cepa and A. fistulosum I. The initiation and sequence of pairing. Chromosoma, 95, 324-338.

ALBINI, S. M. AND JONES, G. H. 1988. Synaptonemal complex spreading in Allium cepa and A. fistulosum II. Pachytene observations. The SC karyotype and the correspondence of late recombination nodules and chiasmata. Genome, in press.

BROWN, S. W. AND ZOHARY, D. 1955. The relationship of chiasmata and crossing-over in Lilium formosanum. Genetics, 40, 850-873.

BURGOYNE, P. S. AND BAKER, T. G. 1984. Meiotic pairing and gametogenic failure. In Evans C. W. and Dickinson, H. G. (eds), Society for Experimental Biology Symposium 38, Company of Biologists, Cambridge.

CARPENTER, A. T. C. 1979. Synaptonemal complex and recombination nodules in wild-type Drosophila melanogaster females. Genetics, 92, 511-541.

CARPENTER, A. T. C. 1984. Recombination nodules and the mechanism of crossing-over in Drosophila. In Evans, C. W. and Dickinson, H. G. (eds), Society for Experimental Biology Symposium 38, Company of Biologists, Cambridge.
CARPENTER, A. T. C. 1987. Gene conversion, recombination nodules, and the initiation of meiotic synapsis Bioessays, $6,232-236$.

EVANS, E. P., BURTENSHAW, M. D. AND CATTANACH, B. M. 1982. Meiotic crossing-over between the $X$ and $Y$ chromosomes of males carrying the sex reversing $(S x r)$ factor. Nature, 300, 443-445.

GLAMANN, J. 1986. Crossing over in the male mouse as analysed by recombination nodules and bars. Carlsberg Res. Commun., 51, 143-162.

HOLM, P. B. AND RASMUSSEN, S. W. 1983. Human meiosis VI. Crossing over in human spermatocytes. Carlsberg Res. Commun, 48, 385-413.

HUltén, M. 1974. Chiasma distribution at diakinesis in the normal human male. Hereditas, 76, 55-78.

JONES, G. H. AND ALBINI, S. M. (In press). Meiotic roles of nodule structures in zygotene and pachytene nuclei of Angiosperms. In Brandham P.E. and Bennet M. D. (eds.) Kew Chromosome Conference III.

KEITGES, E., RIVEST, M., SINISCALCO, M. AND GARTLER, S. M. 1985. X-linkage of steroid sulphatase in the mouse is evidence for a functional-Y-linked allele. Nature 315, 226227.

LOIDL, J. AND GREILHUBER, J. 1983. Structural changes of Ag-NORs and nucleoli during meiosis in Allium flavum. Canad. J. Genet. Cytol, 25, 524-529.

LOIDL, J. AND JONES, G. H. 1986. Synaptonemal complex spreading in Allium I. Triploid A. sphaerocephalon. Chromosoma, 93, 420-428.

MATHER, K. 1935. Reductional and equational separation of the chromosomes in bivalents and multivalents. $J$. Genet., $30,53-78$

ORELlANA, J. AND GIRALDEZ, R. 1981. Metaphase I bound arms and crossing-over frequency in rye. Chromosoma, 84 , 439-449.

RAHN, M. I. AND SOLARI, A. J. 1986. Recombination nodules in the oocytes of the domestic chicken Gallus domesticus. Cytogenet. Cell Genet., 43, 187-198.

ROUYER, F., SIMMLER, M. C., JOHNSSON, C., VERGNAUD, G., COOKE, H. J. AND WEISSENBACH, J, 1986. A gradient of sex linkage in the pseudoautosomal region of the human sex chromosomes. Nature, 319, 291-295.

vosA, C. G. 1976. Heterochromatic patterns in Allium. I. The relationship between the species of the cepa group and its allies. Heredity, 36, 383-392.

WETTSTEIN, D. VON, RASMUSSEN, S. W. AND HOLM, P. B. 1984. The synaptonemal complex in genetic segregation. Ann. Rev. Genet., 18, 331-413.

ZEN, S. 1961. Chiasma studies in structural hybrids. VI. Heteromorphic bivalent and reciprocal translocation in Allium fistulosum. Cytologia, 26, 67-73. 